\title{
EVALUACIÓN DE LA ESTRATEGIA PEDAGÓGICA "APRENDIZAJE BASADO EN PROYECTOS": PERCEPCIÓN DE LOS ESTUDIANTES
}

\author{
EDUARDO RODRÍGUEZ-SANDOVAL* \\ Misael Cortés-RodrigueZ**
}

Recebido em: 27 de agosto de 2009

Aprovado em: 16 de setembro de 2009

*PhD.en Ingeniería, Universidad del Valle. Docente Asociado del Departamento de Ingeniería Agrícola y Alimentos. Director del Laboratorio de Procesos Agrícolas. Facultad de Ciencias Agropecuarias. Universidad Nacional de Colombia, sede Medellín, Antioquia. E-mail: edrodriguezs@unal.edu.co.

${ }^{* *} \mathrm{PhD}$ en Ciencia y Tecnología de Alimentos, Universidad Politécnica de Valencia. Docente Asociado del Departamento de Ingeniería Agrícola y Alimentos. Director del Grupo de Alimentos Funcionales (GAF). Facultad de Ciencias Agropecuarias. Universidad Nacional de Colombia, sede Medellín, Antioquia. E-mail: mcortesro@unal.edu.co

Resumen: El propósito de este trabajo es mostrar la percepción que tienen los estudiantes del programa de Ingeniería Agrícola de la Universidad Nacional de Colombia, sede Medellín sobre la evaluación y el desarrollo de la estrategia pedagógica "aprendizaje basado en proyectos", utilizada en diferentes cursos teórico-prácticos. Esta estrategia consiste en adquirir el aprendizaje del curso por medio de la elaboración y desarrollo de un proyecto durante el semestre académico. El proyecto contribuye a la transferencia del conocimiento de los principios teóricos presentados en clase, al desarrollo del pensamiento crítico y reflexivo, e incentiva el interés por la investigación. El análisis de los resultados de la encuesta aplicada a los estudiantes que participaron en esta experiencia pedagógica, mostró que el aprendizaje basado en proyectos es importante para su desarrollo profesional y cumple con sus expectativas educativas. La mayoría de los encuestados afirman que se aplicaron los conceptos vistos en clase durante el desarrollo del proyecto, la metodología propuesta fue la adecuada, y estuvieron de acuerdo con la valoración final del proyecto y el tiempo dedicado a la parte experimental.

Palabras claves: Evaluación. Ingeniería. Formación. Aprendizaje basado en proyectos.

\section{EVALUATION OF THE PEDAGOGICAL STRATEGY "PROJECT-BASED LEARNING": PERCEPTION OF THE STUDENTS}

\begin{abstract}
The purpose of this study is to show the perception of the agricultural engineering students from Universidad Nacional de Colombia on the evaluation and performance of the pedagogical strategy called "project-based learning", involved in different theoretical-practice courses. This model consists of a learning strategy through the implementation of a project during the academic semester. The project contributes to the transfer of theoretical concepts, the development of critical and reflexive thinking, and to the increase of interest in research and investigation. The survey results from the students who participate in this pedagogical experience showed that projectbased learning is important for their professional development and achieves their educational expectations. Most of the students affirmed that the information learned in class was applied during the project development, the methodology was suitable, and the final assessment of the project and the experimental time were adequate.
\end{abstract}

Key words: Assessment. Engineering. Education. Project-based learning. 


\section{INTRODUCCIÓN}

El aprendizaje se puede ver como un proceso acumulativo, auto-regulado dirigido, colaborativo e individual (VAN DEN BERGH et al., 2006). Aprender es poder justificar lo que se piensa con procesos de producción y aceptación de conocimientos que se desarrollan en la vida cotidiana, los cuales son diferentes a los trabajos científicos. El aprendizaje sólido de los conceptos científicos debe ir acompañado del aprendizaje metodológico, es decir, de formas de producir y recibir conocimientos que caracterizan el trabajo científico. (BECERRALABRA et al., 2007)

Diferentes experiencias y estrategias de enseñanza-aprendizaje han cambiado el papel que había desempeñado un estudiante de receptor de conocimiento pasivo a ser activo, el cual tiene pensamiento crítico con los conocimientos adquiridos dentro y fuera del aula (REITMEIER, 2002). En estudios realizados se ha comprobado que la retención del conocimiento adquirido después de $24 \mathrm{~h}$ en un estudiante es de 5\% para clases magistrales, $50 \%$ para discusión en grupo, $75 \%$ para experiencias prácticas y $90 \%$ por enseñar a otros. (SOUSA, 1995)

La función primordial de las instituciones de educación superior, en particular en áreas de ciencia e ingeniería, es la formación de profesionales cuyo ejercicio se base en el espíritu y método científico, en valores de convivencia, con una sólida capacidad para aprender, característica indispensable en una sociedad en permanente cambio. En la actualidad, la sociedad requiere de un ingeniero innovador, audaz en la experimentación, con habilidades de interacción y de intercambio de ideas con otros profesionales de diferentes áreas (DUQUE; MARTÍNEZ, 2000). Lo anterior implica el establecimiento de una sólida comunidad académico-cultural, que rompa con los esquemas mentales que generan una presunta separación entre saber científico y saber humanístico. De nada sirve formar ingenieros académicos ajenos a la sensibilidad humana, así como tampoco vale la pena graduar artistas sin ningún asomo de rigor científico.

La ingeniería es la conceptualización, diseño, construcción y administración de proyectos y productos orientados a dar solución a una necesidad de la sociedad o del entorno. Por esta razón, el ingeniero debe resolver problemas o proveer diferentes soluciones, lo cual requiere de imaginación, creatividad y síntesis de conocimientos (DUQUE et al., 1999). La ingeniería, en general, es un proceso de toma de decisiones para la solución de problemas dentro de un campo particular de acción. Esta toma de decisiones implica diferentes pasos, entre los cuales se destacan: Delimitar la situación, plantear una estrategia 
de solución, obtener información experimental o teórica, analizar los datos y resultados, seleccionar los criterios valorativos sobre las posibles soluciones, elegir la variable óptima y corregir la decisión durante su implementación. (GARZA-RIVERA, 2001)

En línea con una rápida evolución hacia una sociedad de conocimiento global, el mercado de trabajo contemporáneo demanda profesionales con nuevos conocimientos y capacidades. En la actualidad, el éxito en el campo laboral implica una capacidad para actuar y proponer soluciones en ambientes cambiantes y poco definidos, interactuar en situaciones no rutinarias, sintetizar procesos de trabajo, tomar decisiones responsables y trabajar en equipo. Por lo tanto, los estudiantes universitarios necesitan adquirir no solo la conceptualización en su disciplina, sino también una alta destreza específica en su campo de acción, así como habilidades, actitudes y aptitudes. Cualquier currículo universitario requiere desarrollarse teniendo como base la preparación de los estudiantes para un futuro, que en la mayoría de los casos es desconocido. En este ambiente dinámico para la educación superior se impone una revisión crítica de la enseñanza tradicional y de las prácticas de aprendizaje. (VAN DEN BERGH et al., 2006)

Los profesionales como fuerza laboral necesitan ser diligentes en la solución de problemas, en la interacción con clientes, en la realización de presupuestos, y en el manejo adecuado del tiempo, sin olvidar la competencia responsable frente a las otras compañías. La medida real de la educación se refleja en lo que hacen los estudiantes con lo que han aprendido. El aprendizaje basado en proyectos (ABPr) parece ser un método de enseñanza efectivo comparado con las estrategias de enseñanza cognitivas tradicionales, particularmente para el desarrollo de habilidades en la solución de problemas de la vida real. (WILLARD; DUFFRIN, 2003)

La aproximación al aprendizaje que incluye el trabajo para una solución a un problema se denomina frecuentemente "aprendizaje basado en problemas" (ABP) (METTAS; CONSTANTINOU, 2007). En el ABP se propone a los estudiantes un problema para ser solucionado y aprender más acerca de este, por medio de un trabajo grupal o un estudio independiente (MAUDSLEY, 1999; RESTREPO-GÓMEZ, 2005). Con esta estrategia, el aprendizaje es propositivo y autónomo, ya que el estudiante aprende a medida que investiga las soluciones a los problemas que se han formulado. Algunos aspectos del ABP se fundamentan en problemas intencionalmente mal estructurados (illstructured), situaciones auténticas, y oportunidades para desarrollar autonomía y responsabilidad. (ARAZ; SUNGUR, 2007) 
El tipo de aprendizaje a través de la implementación de proyectos se denomina "aprendizaje basado en proyectos" (ABPr). Con la aplicación de esta estrategia, los estudiantes definen el propósito de la creación de un producto final, identifican su mercado, investigan la temática, crean un plan para la gestión del proyecto, diseñan y elaboran un producto. Los estudiantes comienzan el proyecto solucionando problemas hasta llegar a su producto. El proceso completo es auténtico, referido a la producción en forma real, utilizando las propias ideas de los estudiantes y completando las tareas en la práctica (METTAS; CONSTANTINOU, 2007). Dentro de este marco, los estudiantes persiguen soluciones a problemas no triviales, generando y refinando preguntas, debatiendo ideas, realizando predicciones, diseñando planes y/o experimentos, recolectando y analizando datos, estableciendo conclusiones, comunicando sus ideas y resultados a otros, realizando nuevas preguntas y creando o mejorando productos y procesos. (BLUMENFELD et al., 1991)

En la práctica, la línea de división entre el ABP y el ABPr generalmente se distorsiona. Los dos se utilizan en forma combinada y juegan papeles complementarios (METTAS; CONSTANTINOU, 2007), aunque algunos consideran que el ABP es un tipo de aprendizaje basado en proyectos (WATSON, 2002). El ABP y ABPr tienen la misma orientación, ambos son auténticos, utilizan aproximaciones constructivistas para el aprendizaje, se diseñan centrados en el estudiante e incluyen el papel del docente como orientador. En la educación relacionada con la tecnología, las actividades de solución de problemas ofrecen a los estudiantes la oportunidad de buscar, procesar y aplicar conocimiento a través de la experimentación. (METTAS; CONSTANTINOU, 2007)

En la educación basada en proyectos, los docentes necesitan crear espacios para el aprendizaje dando acceso a la información, soportando la enseñanza por la instrucción, modelamiento y guía a los estudiantes para manejar apropiadamente sus tareas, animar a los estudiantes a utilizar procesos de aprendizaje metacongnitivos, respetar los esfuerzos grupales e individuales, verificar el progreso, diagnosticar problemas, dar retroalimentación, y evaluar los resultados generales. Adicionalmente, los docentes necesitan crear un ambiente conductivo con el fin de fomentar la indagación constructiva y asegurar que el trabajo se realice en una forma eficiente y ordenada (BLUMENFELD et al., 1991). Simultáneamente, el docente debe actuar como orientador del aprendizaje y de los procesos, y dejar que los estudiantes adquieran autonomía y responsabilidad en su aprendizaje. (JOHARI; BRADSHAW, 2008)

La innovación educacional se disolverá por si misma cuando la evaluación no sea congruente con los métodos de enseñanza. Es muy importante realizar 
una evaluación acorde con la instrucción y apropiada con lo que los estudiantes aprenden (DOCHY; MC DOWELL, 1997; SEGERS et al., 2006; BOUSSADA; DE KETELE, 2008). Las evaluaciones pueden ser de varios tipos entre las que se destacan la evaluación de pares, el cual los grupos o cada estudiante evalúan su par, la co-evaluación o evaluación colaborativa, donde tanto los estudiantes como los profesores participan en los procesos de calificación, la evaluación de desempeño, en el cual los estudiantes se evalúan por la forma como solucionan un problema auténtico y real, y el sistema de reportes, donde se utilizan documentos o avances para observar el progreso de los estudiantes en los procesos de aprendizaje. (VAN DEN BERGH et al., 2006)

El estudio de diferentes sistemas de evaluación en el ABPr fue realizado por Van den Bergh et al., (2006) y concluyeron que los métodos de evaluación tradicional son los menos indicados para medir el nivel de compresión y habilidad que los estudiantes adquieren a través del ABPr. Los estudiantes y los docentes consideraron que un buen método de evaluación debe cumplir con los siguientes parámetros: La objetividad (que todos los estudiantes tengan las mismas oportunidades), la transparencia (toda la información es clara y accesible a todos los estudiantes) y la estandarización (el docente debe tener la capacidad de justificar y detallar la evaluación realizada). Además, los estudiantes desean no solo una evaluación del producto final de su proyecto, sino que también se verifique el proceso para llegar a este.

El objetivo de este estudio es presentar la percepción que tienen los estudiantes del programa de Ingeniería Agrícola de la Universidad Nacional de Colombia, sede Medellín sobre la forma como se realiza el desarrollo y la evaluación de la estrategia pedagógica denominada "aprendizaje basado en proyectos". Los resultados que se obtengan de este trabajo servirán para replantear y mejorar varios aspectos en el momento de aplicar esta pedagogía en los cursos teórico-prácticos de la Universidad.

\section{METODOLOGÍA}

Estrategia pedagógica: Al comenzar el curso, se les plantea a los estudiantes la ejecución de un proyecto que deben desarrollar a lo largo del semestre académico, en el cual tienen que aplicar los conceptos teóricos vistos en clase. Las opciones de los estudiantes en la selección de dicho proyecto son varias, pueden escogerlo si tienen alguna inclinación por un tema, si están interesados en resolver un problema específico, o también el profesor puede sugerir proyectos de investigación que se están desarrollado en la institución. 
Después de seleccionar la temática, los estudiantes deben tratar de justificar el estudio por medio de información económica, verificando el mercado, la viabilidad y la importancia del proyecto, y si es posible su impacto a nivel local, regional o nacional. A continuación, el grupo de trabajo determina los objetivos del estudio, realiza y organiza la planeación experimental teniendo en cuenta los materiales y los métodos que se van a utilizar, así como las fuentes de información consultadas. Una vez se reúne la información, se debe orientar al equipo de trabajo. El profesor desempeña un papel de orientador dando recomendaciones, formulando interrogantes, ayudando al grupo en el proceso de toma de decisiones y permitiendo que los estudiantes desarrollen su tarea de forma independiente. Posteriormente se realizan pruebas preliminares para ajustar la metodología y los objetivos propuestos, y después se organiza y ejecuta la experimentación final del proyecto.

El grupo de trabajo de cada proyecto planea, organiza, realiza la experimentación, y presenta en forma escrita y oral los resultados. Los grupos están conformados por 2 o 3 estudiantes, dependiendo del proyecto escogido y el número de estudiantes en cada curso. La evaluación del proyecto se realiza por medio de trabajos escritos y orales así: 1) La propuesta inicial con justificación, 2) el planteamiento de objetivos y metodologías, 3) trabajos escritos parciales de las pruebas experimentales, 4) presentación oral final y 5) reporte escrito final del proyecto; estos dos últimos son los que tienen mayor valor porcentual porque es el resultado general de toda la vivencia experimental del semestre. El formato del reporte escrito final sigue la presentación de un trabajo científico normal constituido por título, autores, resumen, introducción, objetivos, materiales y métodos, resultados y discusión, conclusiones y referencias, con tablas, gráficos y figuras.

Evaluación de la Estrategia Pedagógica: La evaluación de la estrategia pedagógica ABPr se llevó a cabo aplicando un cuestionario (Tabla 1), el cual fue elaborado por un grupo de trabajo de la Universidad, en donde se indagó sobre la percepción que tienen los estudiantes en la forma como se desarrolla y evalúa el ABPr.

Esta encuesta fue realizada en Junio de 2009 a 14 estudiantes del programa de Ingeniería Agrícola de las siguientes asignaturas: Transferencia de Calor con Aplicaciones en los Sistemas Agrícolas, y Transferencia de Masa y Manejo de Sólidos con Aplicaciones en los Sistemas Agrícolas. Las preguntas de la encuesta fueron cerradas y se contestaba marcando "SI" y "NO". Además, se dejaba un espacio al final para que el estudiante expresara sus comentarios generales sobre el curso o la estrategia pedagógica utilizada. Los resultados 
se analizaron empleando el programa Excel ${ }^{\circledR}$, que permite realizar estadística descriptiva, con un porcentaje de error del $5 \%$ en el nivel de significancia. (TOVAR-LÁZARO et al., 2005)

\begin{tabular}{|c|c|c|}
\hline Preguntas & Respu & tas \\
\hline $\begin{array}{l}\text { 1. ¿Cree usted qué el proyecto de aula es importante para su forma- } \\
\text { ción profesional? }\end{array}$ & SI_ & NO \\
\hline $\begin{array}{l}\text { 2. ¿Fue difícil encontrar una idea de proyecto apropiado para desar- } \\
\text { rollar en el curso? }\end{array}$ & $\mathrm{SI}$ & NO \\
\hline $\begin{array}{l}\text { 3. ¿Se aplicaron los conceptos vistos en clase para la solución de los } \\
\text { problemas del proyecto de aula? }\end{array}$ & $\mathrm{SI}$ & $\mathrm{NO}$ \\
\hline $\begin{array}{l}\text { 4. ¿Cree usted que la forma de realizar el proyecto durante el semestre } \\
\text { fue la adecuada? }\end{array}$ & SI_ & $\mathrm{NO}$ \\
\hline $\begin{array}{l}\text { 5. ¿Esta conforme con la forma de realizar los avances (entregas) del } \\
\text { proyecto de aula durante el semestre? }\end{array}$ & $\mathrm{SI}$ & $\mathrm{NO}$ \\
\hline $\begin{array}{l}\text { 6. ¿Considera que la valoración final del proyecto corresponda al } 33 \% \\
\text { de la calificación final de la asignatura? }\end{array}$ & SI_ & $\mathrm{NO}$ \\
\hline $\begin{array}{l}\text { 7. ¿El tiempo estipulado para el desarrollo experimental del proyecto } \\
\text { es suficiente? }\end{array}$ & SI_ & $\mathrm{NO}$ \\
\hline $\begin{array}{l}8 \text { ¿Este tipo de metodologías para el aprendizaje satisfacen sus expec- } \\
\text { tativas como estudiante comparándola con otros métodos tradicionales? }\end{array}$ & SI_ & $\mathrm{NO}$ \\
\hline $\begin{array}{l}9 \text { ¿Considera que los materiales, equipos de laboratorios y demás } \\
\text { recursos disponibles actualmente, son los adecuados para el desarrollo } \\
\text { de la parte experimental del proyecto? }\end{array}$ & SI_ & $\mathrm{NO}$ \\
\hline $\begin{array}{l}10 \text { Usted considera que la orientación para el desarrollo del trabajo } \\
\text { por parte del docente cumplió con sus expectativas? }\end{array}$ & SI_ & $\mathrm{NO}$ \\
\hline
\end{tabular}

Tabla 1 - Encuesta realizada a los estudiantes para conocer su percepción sobre el desarrollo y evaluación de la estrategia pedagógica ABPr.

\section{RESULTADOS Y DISCUSIÓN}

El resultado de las primeras cinco (5) preguntas de la encuesta se recopilaron en la Figura 1. En la primera pregunta todos los estudiantes consideraron que la estrategia pedagógica $\mathrm{ABPr}$ es un apoyo importante en su desarrollo profesional. Un número de factores deberían ser considerados en el diseño de un ABPr para motivar a los estudiantes en el desarrollo y ejecución del proyecto (BLUMENFELD et al., 1991), siendo los más relevantes la comprensión de lo 
interesante y valioso que puede ser un proyecto, la competencia para completar el proyecto, y el enfoque en el aprendizaje más que en los resultados y las calificaciones. Además, también se debe tener una experiencia o práctica real debido a que la mayoría de los estudiantes de ingeniería van a formar parte del sistema productivo. El sistema educativo actual generalmente se dirige hacia los logros individuales, pero la regla en la industria moderna es trabajar como equipo, donde un logro individual frecuentemente se cuenta como productivo para las metas del grupo. (ALPTEKIN et al., 2005; BOURGEON, 2007)

En la segunda pregunta, el 57\% de los encuestados considero que fue difícil encontrar una idea de proyecto para desarrollar en el curso. Este resultado refleja la falta de autonomía de muchos estudiantes para desarrollar un proyecto de acuerdo a sus inclinaciones académicas o profesionales, además, es importante resaltar que los estudiantes deben escoger una temática al inicio del curso sin tener claro los recursos con los que se dispone y los conceptos teóricos que se van a tocar en el curso. Esta situación crea un poco de confusión en los estudiantes al momento de seleccionar un tema. Existen dos componentes esenciales de los proyectos, el primero se refiera a la formulación de una pregunta o problema que sirva para organizar y direccionar las actividades, y el segundo consiste en que dichas actividades resultan en una serie de productos o procesos, que culminan en un producto/proceso final que dirigen las preguntas. (BLUMENFELD et al., 1991)

El sistema educativo tradicional en ingeniería ha condicionado permanentemente a los estudiantes en el desarrollo de problemas y ejercicios de la literatura, sin prepararlos para problemas o situaciones reales, lo cual tiene más sentido si se considera que en un futuro van a formar parte de un sector productivo con repercusiones directas sobre la sociedad. Los estudiantes que entran en el sistema de educación basado en proyectos con más autonomía, tienden a tener experiencias más positivas y a percibir mejor este tipo de aprendizaje. Es importante que los profesores se esfuercen y estimen un buen tiempo al comienzo del curso en la explicación de la dinámica de esta estrategia pedagógica. Si los estudiantes están comprometidos y asumen que este tipo de aprendizaje es importante por la valiosa experiencia profesional y el desarrollo de habilidades, tendrán una actitud y disposición favorable para afrontar el proyecto durante el curso. (LIU et al., 2008)

En la pregunta 3 del cuestionario, se indagaba por la aplicación de los conocimientos vistos en clase para la solución de los problemas del proyecto, y solo el 7\% de los encuestados respondieron negativamente. El ABPr sitúa a los estudiantes en ambientes reales y contextualizados, y puede servir para 
construir puentes entre los conceptos teóricos de las temáticas del curso y las experiencias de la vida real. Además, el ABPr promueve los lazos entre diferentes disciplinas o áreas de conocimiento y se adapta a diferentes tipos de estudiantes y situaciones de aprendizaje. (BLUMENFELD et al., 1991)

La forma como se desarrolló el ABPr durante el curso se examinó en la pregunta 4. La mayoría de los estudiantes (71\%) consideraron que el ABPr se aplicó en forma apropiada durante el curso; sin embargo, otros estudiantes (29\%) contestaron negativamente. Esto último puede explicarse por el número de entregas o avances del proyecto que se realizaron durante el semestre, pues varios manifestaron la necesidad de incrementar este tipo de trabajos para afianzar y mejorar el desarrollo experimental del proyecto. Con respecto a este tema es complicado aumentar el número de avances porque la carga de trabajo para el docente y las diferencias entre los diversos proyectos limitan un poco la gestión, organización y evaluación de esta estrategia pedagógica. (VAN DEN BERGH et al., 2006)

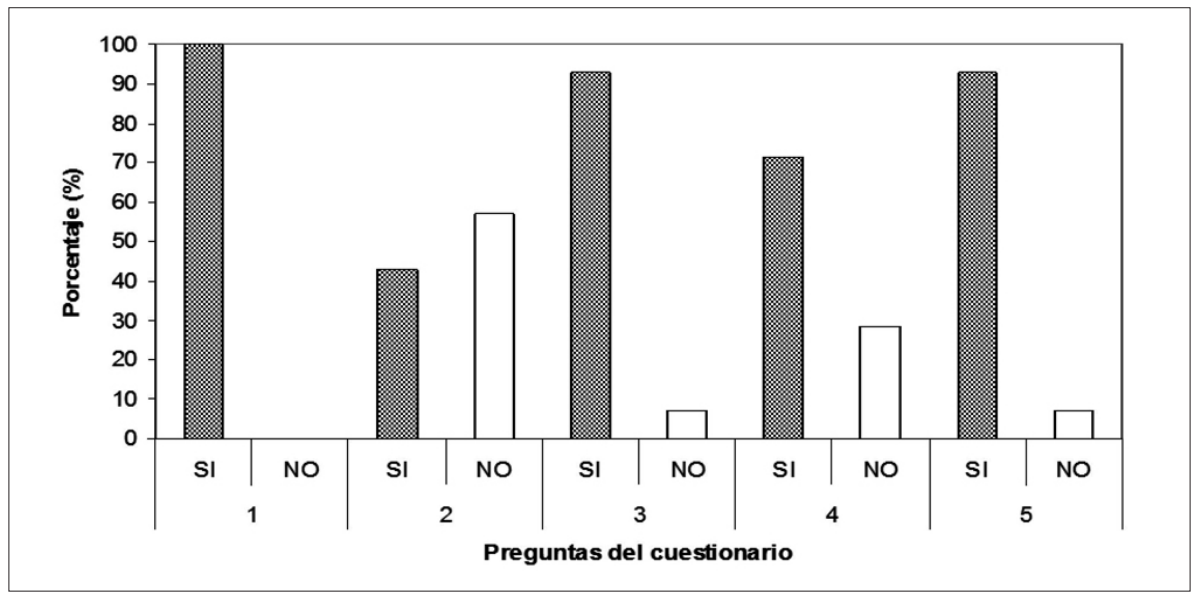

Figura 1 - Resultados de la pregunta 1 a la 5 del cuestionario aplicado a los estudiantes del programa en Ingeniería Agrícola.

La pregunta 5 se relaciona con la apreciación sobre la realización de los avances (entregas) del proyecto durante el semestre. E1 93\% de los encuestados aprobó este tipo de metodología. Los informes que presentaban los estudiantes a medida que avanzaban en el proyecto demostraron como se mejoraba la capacidad para sintetizar y analizar los resultados que obtenían parcialmente. 
Después de la experiencia práctica, el conocimiento y la teoría aplicada a cada proyecto se evidenciaba en las exposiciones y trabajos finales de los estudiantes.

En la Figura 2 se muestran los resultados de la pregunta 6 a la 10 de la encuesta aplicada a los estudiantes. En la pregunta 6 se investigó la percepción que tienen los estudiantes sobre la valoración del trabajo final (exposición oral y trabajo escrito) del proyecto con respecto al porcentaje total de la evaluación del curso que corresponde a un 33\%. El 79\% de los encuestados esta de acuerdo con dicha valoración, mientras que el resto (21\%) desaprueba el valor propuesto para el proyecto del curso. La aplicación del ABPr en una asignatura es una herramienta para mejorar la comunicación entre el grupo del proyecto y el resto del grupo de clase o los pares evaluadores (MURPHY; GAZI, 2001), siendo el documento escrito y la exposición oral del proyecto esenciales para comprobar el cumplimiento de estas competencias al final del curso.

La prueba típica estandarizada o las preguntas de un texto guía enfocadas en un nivel bajo de comprensión es inapropiado para examinar los beneficios de la instrucción basada en proyectos. Ejemplos de medidas informales que pueden dar una guía y retroalimentación tanto para los profesores como para los alumnos son documentos de avance, evaluación de portafolio, entrevistas clínicas, y exposiciones de los estudiantes. Sin embargo, el profesor debe tener cuidado en la utilización de estas técnicas para diagnosticar la comprensión del estudiante debido a que son menos estructuradas, más clínicas, y consumen más tiempo. (BLUMENFELD et al., 1991)

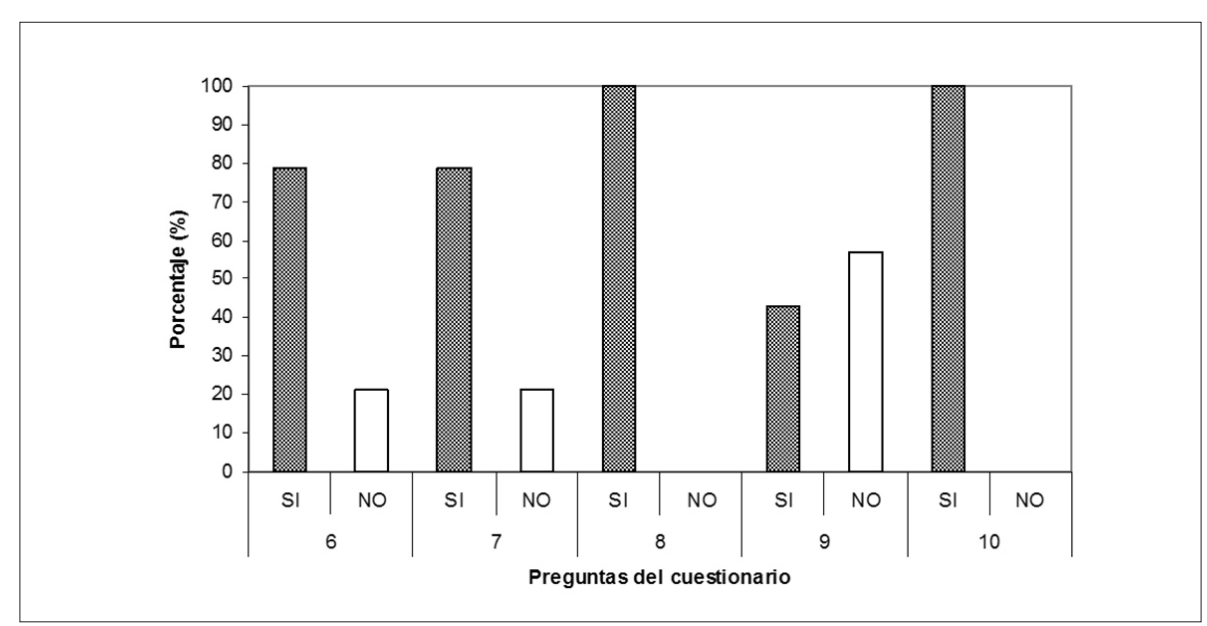

Figura 2 - Resultados de la pregunta 6 a la 10 del cuestionario aplicado a los estudiantes del programa en ingeniería agrícola. 
La evaluación del tiempo para el desarrollo experimental del proyecto se determinó en la pregunta 7. La mayoría de los estudiantes (79\%) consideraron que el tiempo para el desarrollo de la parte práctica del proyecto fue suficiente; no obstante, los encuestados que respondieron negativamente $(21 \%)$ argumentan que el tiempo destinado al laboratorio no fue lo suficiente por los problemas logísticos que tuvieron. Los inconvenientes que se presentan durante el desarrollo del proyecto se utilizan como herramientas para adquirir el conocimiento y la habilidad para solucionar problemas. Lo esencial es que los estudiantes aprendan a analizar e interpretar datos experimentales con el fin de solucionar problemas representativos, siendo este el corazón del método. (DOCHY et al., 2003)

El ABPr se enfoca en el desarrollo de las siguientes habilidades en los estudiantes: Formular preguntas de investigación, proponer problemas, escoger una pregunta dirigida, escribir una propuesta, afrontar una evaluación de un par, desarrollar criterios de evaluación y métodos de investigación y desarrollo, analizar y registrar datos, y establecer conclusiones (FALLIK et al., 2008). En el mejoramiento de las relaciones entre la motivación y el pensamiento se ha recurrido con frecuencia a la idea de proyectos, donde se realizan en un tiempo determinado, con problemas enfocados y unidades de instrucción que integran conceptos de un número de disciplinas o campos de estudio. (BLUMENFELD et al., 1991)

La comparación entre la metodología de enseñanza tradicional y el ABPr se llevo a cabo en la pregunta 8 . Todos los estudiantes consideran que la educación con ABPr satisfacen sus expectativas de educación y en diversos comentarios resaltan la importancia de esta estrategia pedagógica. Varios trabajos han demostrado que los estudiantes en un ABPr presentan mejores calificaciones en las pruebas de logros que los alumnos en un grupo normal (control), además desarrollan habilidades de aprendizaje independiente (incluyendo la solución de problemas), aprenden a tener una mente abierta y recuerdan e interiorizan lo aprendido por más tiempo (MIODUSER; BETZER, 2007; FALLIK et al., 2008). Los proyectos deben ser un reto para los estudiantes, pero sin ser muy complejos. Esta combinación de características conduce a encontrar actividades lúdicas en el proyecto. Los problemas requieren un balance de habilidades y desafíos que puedan generar una experiencia agradable en el aprendizaje. (JOHARI; BRADSHAW, 2008)

Los estudiantes que trabajan en pequeños grupos logran un mayor rendimiento, que cuando se emplean otros métodos de enseñanza. Adicionalmente, tienen mayor habilidad para el razonamiento y el pensamiento crítico, compren- 
sión más profunda de la materia, menores niveles de estrés y ansiedad, mayor motivación, mayor habilidad para ver situaciones desde otras perspectivas, relación más positiva y de apoyo mutuo con compañeros, una mejor actitud hacia la materia y mayor autoestima (MARTÍNEZ-RODRIGO et al., 2007). Sin embargo, a veces se tienen ciertos inconvenientes porque algunos estudiantes no colaboran activamente en el grupo, pero ganan todos los beneficios de la evaluación por el trabajo del equipo en el proyecto (MOESBY et al., 2006). Esto se podría solucionar proponiendo diferentes métodos de evaluación para este tipo de aprendizaje.

En la pregunta 9 se evaluaron los recursos disponibles en la Universidad para realizar la parte experimental del proyecto. El 57\% de los estudiantes consideró que las condiciones de los equipos de los laboratorios no son los más apropiados para desarrollar la parte experimental del proyecto. Durante el semestre académico, se presentaron inconvenientes logísticos con ciertos equipos para desarrollar las prácticas de laboratorio del proyecto, pero como se mencionó anteriormente, esto se puede utilizar como herramientas para mejorar la habilidad y la capacidad en la solución de problemas. En ningún lugar se va a disponer de todos los recursos para desarrollar un proyecto, y es en este punto donde la creatividad, el ingenio y la imaginación del grupo de trabajo deben sobresalir para afrontar este tipo de situaciones.

En la pregunta 10 se evaluó el trabajo realizado por el profesor durante la estrategia pedagógica de ABPr. Nuevamente todos los estudiantes aprobaron la gestión realizada por el docente en el desarrollo del proyecto planteado en el curso. Las nuevas aproximaciones cognitivas que representan los proyectos requieren de un cambio sustancial del pensamiento y disposición de los profesores hacia las actividades, tareas y la estructura de la clase. (BLUMENFELD et al., 1991)

\section{CONCLUSIONES}

La encuesta realizada para verificar la forma como se aplica y evalúa el aprendizaje basada en proyectos (ABPr) en diferentes asignaturas del programa de Ingeniería Agrícola de la Universidad Nacional de Colombia, sede Medellín, presenta un panorama general de la visión y la percepción de los estudiantes frente a esta estrategia pedagógica de aprendizaje. Todos los estudiantes resaltan la importancia de este tipo de estrategias para su formación profesional y consideran que el ABPr cumple con sus expectativas de educación. Además, la mayoría de los encuestados afirman que se aplicaron los conceptos vistos en 
clase durante el desarrollo del proyecto (93\%), fue adecuado la forma de realizar el proyecto (71\%), fue acertado los avances entregados (93\%), estuvieron de acuerdo con la valoración final del proyecto (79\%) y el tiempo estimado para el desarrollo experimental (79\%). Por otro lado, los estudiantes estimaron que fue difícil encontrar una idea de proyecto apropiada para realizar en el curso $(57 \%)$ y que los recursos físicos no fueron los adecuados para afrontar la parte experimental del proyecto (57\%). La integración entre la metodología, la evaluación y las actividades de orientación en el ABPr son necesarias en el fortalecimiento de la pedagogía en los cursos de ingeniería. La percepción de los estudiantes frente a este tipo de estrategias pedagógicas es muy buena, aunque se debe tener en cuenta que debe existir un balance adecuado entre el ambiente de aprendizaje y la evaluación de esta propuesta educativa.

\section{AGRADECIMIENTOS}

Los autores quieren agradecer a los estudiantes del programa de Ingeniería de Agrícola de la Universidad Nacional de Colombia, sede Medellín que participaron en la encuesta realizada en Junio del 2009.

\section{REFERENCIAS}

ALPTEKIN, S.E.; DETURRRIS, D.; MACY, D.J.. Development of a flying eye: A project-based learning experience. Journal of Manufacturing Systems, Michigan, EE.UU, v. 24, n. 3, p. 226-236, 2005

ARAZ, G.; SUNGUR, S. The interplay between cognitive and motivational variables in a problem-based learning environment. Learning and

Individual Differences, Massachusetts, EE.UU , v. 17, p. 291-297, 2007.

BECERRA-LABRA, C.; GRAS-MARTÍ, A.; MARTÍNEZ-TORREGROSA, J. La física con una estructurada problematizada: efectos sobre el aprendizaje conceptual, las actitudes e intereses de los estudiantes universitarios. Revista Brasileira de Ensino de Física, São Paulo, Brasil, v. 29, n. 1, p. 95-103, 2007.

BLUMENFELD, P.C.; SOLOWAY, E.; MARX, R.W.; KRAJCIK, J. S.; GUZDIAL, M.; PALINCSAR, A. Motivating project-based learning: Sustaining the doing, supporting the learning. Educational Psychologist, Kentucky, EE.UU, v. 26, n. 3 \& 4, p. 369-398, 1991. 
BOURGEON, L. Staffing approach and conditions for collective learning in project teams: The case of new product development projects. International Journal of Project Management, Nijkerk, The Netherlands, v. 25, p. 413-422, 2007.

BOUSSADA, H., DE KETELE, J-M. L'évaluation de la qualité de la formation et du système d'évaluation universitaire: Le point de vue des diplômés. Avaliação: Revista da Avaliação da Educação Superior, Campinas; Sorocaba, SP, Brasil, v. 13, n. 1, p. 39-61, 2008.

DOCHY, F., MCDOWELL, L. Introduction assessment as a tool for learning. Studies in Educational Evaluation, Kiel, Germany, v. 23, n. 4, p. 279-298, 1997

DOCHY, F.; SEGERS, M.; VAN DEN BOSSCHE, P.; GIJBELS, D. Effects of problem-based learning: a metaanalysis. Learning and Instruction, v.13, p. 533-568, 2003.

DUFFRIN, M.W. Integrating problem-based learning in an introductory college food science course. Jounal of Food Science Education, Illinois, EE.UU, v. 2, p. 2-6, 2003.

DUQUE, M.; GAUTHIER, A.; GÓMEZ, R.; LOGUERRERO, J.; PINILLA, A. Formación de ingenieros para la innovación y el desarrollo tecnológico en Colombia. Revista DYNA, Medellín, Colombia, v. 128, p. 63-82, 1999

DUQUE, M.; MARTÍNEZ, A. C. Aprender haciendo: una experiencia de un laboratorio diferente.In: REUNIÓN NACIONAL DE FACULTADES DE INGENIERÍA, 10., 2000, Cartagena de Indias. Ingeniería y Desarrollo Social. Cartagena de Indias: Asociación Colombiana de Facultades de Ingeniería - ACOFI, 2000. p. 14-20.

FALLIK, O., EYLON, B.S., ROSENFELD, S. Motivating teachers to enact free-choice project-based learning in science and technology (PBLSAT): Effects of a professional development model. Jounal of Science Teacher Education, Pennsylvania, EE.UU, v. 19, p. 565-591, 2008.

GARZA-RIVERA, R. G. El rol de la física en la formación del ingeniero. Ingenierías, Nuevo Leon, México, v. 4, n. 13, p. 48-54, 2001. 
JOHARI, A., BRADSHAW, A.C. Project-based learning in an internship program: A qualitative study of related roles and their motivational attributes. Educational Technology Research and Development, Massachusetts, EE.UU , v. 56, p. 329-359, 2008.

LIU, W.C., WANG, C.K.J., TAN, O.S., KOH, C., EE, J. A self-determination approach to understanding students' motivation in project work. Learning and Individual Differences. Doi:10.1016/j.lindif.2008.07.002, 2008.

MARTÍNEZ-RODRIGO, F; HERRERO DE LUCAS, L.C; GONZÁLEZ DE LA FUENTE, J.M; DOMINGUEZ-VÁZQUEZ, J.A. Project based learning experience in industrial electronics and industrial applications design. Universidad de Valladolid. Escuela Universitaria Politécnica, 2007. Disponible en: <http://www.greidi.uva.es/articulos/EUP_ProjectBased. pdf. $>$ Acceso en 11 nov. 2008.

MAUDSLEY G. Do we all mean the same thing by "problem-based learning"? A review of the concepts and a formulation of the ground rules. Academic Medicine, Pennsylvania, EE.UU, v. 74, p. 178-85, 1999.

METTAS, A.C., CONSTANTINOU, C.C. The technology fair: a projectbased learning approach for enhancing problem solving skills and interest in design and technology education. International Journal of Technology and Design Education, Dordrecht, The Netherlands, v. 18, p. 79-100, 2007.

MILENTIJEVIC, I., CIRIC, V., VOJINOVIC, O. Version control in projectbased learning. Computers \& Education, California, EE.UU, v. 50, p. 1331-1338, 2008.

MIODUSER, D., BETZER, N. The contribution of Project-based-learning to high-achievers' acquisition of technological knowledge. International Journal of Technology and Design Education, Dordrecht, The Netherlands, v. 18, p. 59-77, 2007.

MOESBY, E., JOHANNSEN, H.H.W., KØRNØV, L. Individual activities as an integrated part of project work: an innovative approach to project oriented and problem-based learning (POPBL). World Transactions on Engineering and Technology Education, Victoria, Austalia, v. 5, n. 1, p. 11-17, 2006. 
MURPHY, K.L., GAZI, Y. Role plays, panel discussions and simulations: Project-based learning in a web-based course. Educational Media International, Vienna, Austria, v. 38, n. 4, p. 261-269, 2001.

REITMEIER, C.A. Active learning in the experimental study of food. Jounal of Food Science Education, Illinois, EE.UU, v. 1, p. 41-44, 2002.

RESTREPO-GÓMEZ, B. Aprendizaje basado en problemas (ABP): una innovación didáctica para la enseñanza universitaria. Educación y Educadores, Bogotá, Colombia, v. 8, p. 9-19, 2005.

SEGERS, M., NIJHUIS, J., GIJSELAERS, W. Influence on students' perceptions of assessment demands and their learning strategies. Studies in Educational Evaluation, Kiel, Germany, v. 32, p. 223-242, 2006

SOUSA, D.A. How the Brain Learns. Reston, VA: The National Association of Secondary School Principals, 1995.

TOVAR-LÁZARO, M.T., AZÚA-ROMERO, J., GIMÉNEZ-MORALES, J.C. Encuesta a pacientes con baja laboral y análisis estadístico de los resultados. ¿Se puede detectar la simulación a través de encuestas? Revista Medicina General. Sociedad Española de Medicina General (SEMG), Madrid, España, v. 73, p. 215-219, 2005.

VAN DEN BERGH, V., MORTERMANS, D., SPOOREN, P., VAN PETEGEM, P., GIJBELS, D., VANTHOURNOUT, G. New assessment modes within project-based education - the stakeholders. Studies in Educational Evaluation, Kiel, Germany, v. 32, p. 345-368, 2006.

WATSON, G. Using technology to promote success in PBL courses. The technology source. University of Noth Carolina. 2002. Disponible en: http:// technologysource.org/article/using_technology_to_promote_success_in pbl_courses/, May/June. Acceso en: 8 de dic. 2008

WILLARD, K., DUFFRIN, M. W. Utilizing project-based learning and competition to develop student skills and interest in producing quality food items. Jounal of Food Science Education, Illinois, EE.UU, v. 2, p. 69-73, 2003. 